\title{
The mysteries of S6K2 may shed light to breast cancer therapy path.
}

\author{
Nurettin İlter Sever* \\ Department of Molecular Biology and Genetics, Pamukkale University, Denizli, Turkey
}

\begin{abstract}
The divergence between S6 Kinase 2 (S6K2) and its homologue S6 Kinase 1 (S6K1) has displayed that the exclusive functions of $\mathbf{5 6 K 2}$ are very important mediators of tumor growth. Recent studies suggest that S6K2 complexes with B-Raf and PKCE to exert cancer cell survival. Also, indirect roles of S6K2 which involve interaction with Akt and PDCD4 to propagate cancer cell survival make it an important therapeutic target. Also, centrosomal localization of a pool of $56 \mathrm{~K} 2$ potentiates a proliferative role. Amplification and overexpression of RPS6KB2 gene locus, which encodes S6K2 protein, is observed in breast cancer and is correlated with poor prognosis. Also, S6K2 expression is correlated with 4EBP1 and E2F1 expression in breast cancer. Also, breast cancer tissues display nuclear over-accumulation of S6K2 when compared to its normal counterparts. Currently, the mechanisms which regulate the cellular levels of S6K2 are unknown. Also, there still remains new substrates of S6K2 to be unraveled. As the mysteries of $\mathrm{S6K2}$ is solved, new stones are paved in the breast cancer therapy path.
\end{abstract}

Keywords: S6 Kinase 2, Breast cancer, Cancer therapy.

Accepted on 09 April 2019

\section{Introduction}

S6 Kinase family proteins are very important downstream effectors of PI3K/Akt/mTOR signaling pathway. S6K2, as the younger member of S6 Kinase family, has recently gained remarkable attention as its substrates continuously diverge from that of S6K1, the older member of S6 Kinase family. It has two isoforms: p54 and p56. p56 mainly localizes in nucleus whereas p54 displays both nuclear and cytoplasmic localization. Their only common substrates are S6 and PDCD4. S6K1 and S6K2 phosphorylate different substrates and therefore play important roles in cell growth, proliferation, survival and metabolism.

Although most of the structures and the activation patterns of S6K1 and S6K2 are quite similar, recent studies have uncovered exclusive roles for S6K2. The presence of prolinerich domain in S6K2 enlarges its repertoire of substrates, several of which are still unknown [1-3].

The studies in the last two decades have unraveled the link between S6K2 and cancer. Two studies by Pardo et al. [4,5] displayed that S6K2 both induced proliferation and evaded apoptosis in FGF-2 induced small cell lung cancer (SCLC) cells. A very recent study from Amaral et al. [6] unraveled that $\mathrm{S} 6 \mathrm{~K} 2$ promoted cell survival, migration and tumorigenesis in PC3 prostate cancer cell line.

Moreover, more abundant number of studies came from breast cancer. We aim to review the recent breakthroughs that explores the role of S6K2 in breast cancer and to shed light to putative therapeutic approaches.

\section{S6K2 and Breast Cancer}

A Ukranian research group examined the expression pattern of S6K2 in breast cancer in early 2000s. They discovered the overexpression of $\mathrm{S} 6 \mathrm{~K} 2$ in breast cancer tissues when compared to their normal counterparts [7]. In normal breast tissues, S6K2 tended to localize in the cytoplasm whereas it displayed more nuclear pattern in breast cancer tissues [8].

S6K2 expression also displayed positive correlation with PCNA and Ki-67 expression. This might suggest that S6K2 is involved in breast cancer cell proliferation [9]. These characteristics were not observed for S6K1.

A Swedish research group investigated the relationship between S6K2 and breast cancer survival last decade. An earlier study unraveled the amplification of S6K2-encoding RPS6KB2 gene in breast cancer tissues [10]. Positive correlation of S6K2 expression with 4EBP1, another mTOR effector protein which promotes protein translation thus cell growth, was observed in breast tumor tissues from postmenapousal women. Also, higher expression of S6K2 and 4EBP1 yielded lower survival of these women [11]. In both studies, it was displayed that the ER/PgR subgroups and the subcellular localization of both S6K2 and 4EBP1 defined important prognostic values in response to tamoxifen treatment. More cytoplasmic 4EBP1 localization predicted a worse outcome in $\mathrm{ER}+/ \mathrm{PgR}+$ breast cancer subgroup when treated with tamoxifen. On the other hand, nuclear S6K2 predicted a better outcome in $\mathrm{ER}+/ \mathrm{PgR}+$ subgroup, but a worse outcome in $\mathrm{ER}+/ \mathrm{PgR}$ - subgroup in breast cancer cohorts in response to tamoxifen treatment $[10,11]$. Another study by the same group investigated the correlation patterns of S6K1-, S6K2- and 4EBP1-expressing breast tumors with other genes. Interestingly, the correlation between $\mathrm{S} 6 \mathrm{~K} 2$ and 4EBP1 is much more prominent than these two proteins with S6K1 
separately. S6K2 and 4EBP1 displayed very high correlation with cell cycle associated genes, especially with E2F1 and CCNB1 [12].

Two studies by Sridharan and Basu discovered how S6K2 contributed to cancer cell survival in breast cancer cell lines. They found out that downregulation of S6K2 yielded an increase in TNF-induced apoptosis in MCF-7 cells. This induction of apoptosis was overturned by Akt overexpression, pointing out that a positive feedback mechanism of Akt activation by S6K2 is involved in breast cancer cell survival, on the contrary that Akt is negatively regulated by $\mathrm{S} 6 \mathrm{~K} 1$. Bid, a pro-apoptotic protein was also shown to be interfered by S6K2 to maintain breast cancer cell survival [13]. A more recent study by the same group displayed that $\mathrm{Mcl}-1$, an antiapoptotic protein, was regulated by $\mathrm{S} 6 \mathrm{~K} 2$ to maintain survival in T47D cells. This effect was partly blocked by JNK1, suggesting that S6K2 activated Mcl-1 partly via deactivating JNK1 [14].

\section{Conclusion}

The aforementioned studies in this review, exploring the role of S6K2 in breast cancer, have demonstrated that S6K2 might have a great potential in targeted therapy in breast cancer. As long as the new interacting partners and substrates of S6K2 are uncovered, more targeted approaches of S6K2 interactions will be under spotlight.

\section{Future Perspectives}

- There still remains several questions to be answered for S6K2. As long as the mysteries of S6K2 are solved, new paths for breast cancer therapy will be opened.

- The proline-rich domain found in S6K2 prompts that there are several substrates with which S6K2 might be interacting and which are still unknown.

- The mechanisms which regulate S6K2 cellular steady-state level are not completely known.

- The microRNAs which regulate cellular S6K2 level are unknown. The only discovered microRNA is miR-193a-3p.

- The E3 ubiquitin ligase and DUB enzyme for S6K2 are currently unknown.

- There is no current S6K2-specific inhibitor. Its development might direct breast cancer cells to apoptosis and loss of chemotherapy resistance [15].

\section{References}

1. Saxton RA, Sabatini DM. mTOR Signaling in Growth, Metabolism, and Disease. Cell. 2017;168:960-76.

2. Tavares MR, Pavan ICB, Amaral CL, et al. The S6K protein family in health and disease. Life Sci. 2015;131:1-10.

3. Pardo OE, Seck1 MJ. S6K2: The Neglected S6 Kinase Family Member. Front Oncol. 2013;191.

4. Pardo OE, Arcaro A, Salerno G, et al. Novel cross talk between MEK and S6K2 in FGF-2 induced proliferation of SCLC cells. Oncogene. 2001;20:7658-67.
5. Pardo OE, Wellbrock C, Khanzada UK et al. FGF-2 protects small cell lung cancer cells from apoptosis through a complex involving PKCepsilon, B-Raf and S6K2. EMBO J. 2006;25:3078-88.

6. Amaral CL, Freitas LB, Tamura RE, et al. S6Ks isoforms contribute to viability, migration, docetaxel resistance and tumor formation of prostate cancer cells. BMC Cancer. 2016; 16:602.

7. Savinska LO, Lyzogubov VV, Usenko VS, et al. Immunohistochemical analysis of S6K1 and S6K2 expression in human breast tumors. Exp Oncol. 2004; 26:24-30.

8. Filonenko VV, Tytarenko, R, Azatjan SK, et al. Immunohistochemical analysis of S6K1 and S6K2 localization in human breast tumors. Exp Oncol. 2004;26: 294-9.

9. Lyzogubov V, Khozhaenko Y, Usenko V, et al. Immunohistochemical analysis of $\mathrm{Ki}-67, \mathrm{PCNA}$ and S6K1/2 expression in human breast cancer. Exp Oncol. 2005;27:141-144.

10. Pérez-Tenorio G, Karlsson E, Waltersson MA, et al. Clinical potential of the mTOR targets S6K1 and S6K2 in breast cancer. Breast Cancer Res Treat. 2011;128:713-23.

11. Karlsson E, Pérez-Tenorio G, Amin R, et al. The mTOR effectors 4EBP1 and S6K2 are frequently coexpressed, and associated with a poor prognosis and endocrine resistance in breast cancer: A retrospective study including patients from the randomised Stockholm tamoxifen trials. Breast Cancer Res. 2013;15:96.

12. Karlsson E, Magić I, Bostner J, et al. Revealing Different Roles of the mTOR-Targets S6K1 and S6K2 in Breast Cancer by Expression Profiling and Structural Analysis. PLoS One. 2015;10:e0145013.

13. Sridharan S, Basu A. S6 kinase 2 promotes breast cancer cell survival via Akt. Cancer Res. 2011;71:2590-9.

14. Basu A, Sridharan S. Regulation of anti-apoptotic Bcl-2 family protein $\mathrm{Mcl}-1$ by $\mathrm{S} 6$ kinase 2. PLoS One. 2017; 12:e0173854.

15. Sever NI, Cengiz-Sahin S. S6K2 promises an important therapeutic potential for cancer. Future Oncol. 2019;15:95-102.

\section{*Correspondence to:}

Nurettin İlter Sever

Department of Molecular Biology and Genetics

Pamukkale University

Denizli

Turkey

Tel: +905334296166

E-mail: nsever@pau.edu.tr 